\title{
Network-Assisted Distributed Fairness-Aware Interference Coordination for Device-to-Device Communication Underlaid Cellular Networks
}

\author{
Francis Boabang, ${ }^{1}$ Hoang-Hiep Nguyen, ${ }^{1}$ Quoc-Viet Pham, ${ }^{1}$ and Won-Joo Hwang ${ }^{2}$ \\ ${ }^{1}$ Department of Information and Communication System, Inje University, 197 Inje-ro, Gimhae, Gyeongnam, Republic of Korea \\ ${ }^{2}$ Department of Electronic, Telecommunications, Mechanical and Automotive Engineering, HSV-TRC, Inje University, \\ 197 Inje-ro, Gimhae, Gyeongnam 621-749, Republic of Korea
}

Correspondence should be addressed to Won-Joo Hwang; ichwang@inje.ac.kr

Received 21 May 2016; Accepted 19 December 2016; Published 30 January 2017

Academic Editor: Yuh-Shyan Chen

Copyright ( 2017 Francis Boabang et al. This is an open access article distributed under the Creative Commons Attribution License, which permits unrestricted use, distribution, and reproduction in any medium, provided the original work is properly cited.

\begin{abstract}
Device-to-device (D2D) communication underlaid cellular network is considered a key integration feature in future cellular network. However, without properly designed interference management, the interference from D2D transmission tends to degrade the performance of cellular users and D2D pairs. In this work, we proposed a network-assisted distributed interference mitigation scheme to address this issue. Specifically, the base station (BS) acts as a control agent that coordinates the cross-tier interference from D2D transmission through a taxation scheme. The cotier interference is controlled by noncooperative game amongst D2D pairs. In general, the outcome of noncooperative game is inefficient due to the selfishness of each player. In our game formulation, reference user who is the victim of cotier interference is factored into the payoff function of each player to obtain fair and efficient outcome. The existence, uniqueness of the Nash Equilibrium (NE), and the convergence of the proposed algorithm are characterized using Variational Inequality theory. Finally, we provide simulation results to evaluate the efficiency of the proposed algorithm.
\end{abstract}

\section{Introduction}

Device-to-device (D2D) communication underlaid cellular network is considered a key integration feature in the $3 \mathrm{GPP}$ LTE-A systems [1]. Contrary to traditional cellular network where cellular users (CUs) receive services from the base station, this feature allows two communicating CUs in proximity to reuse the radio resources of other CUs and transmit the signal directly without relaying through the BS [2]. Due to close transmission range and spectrum reuse, this kind of communication can enhance the spectral efficiency and resource utilization. However, D2D transmission introduces two sets of interference in cellular networks, interference to CUs and interference amongst neighbouring D2D pairs on each subchannel. This can deteriorate the performance of the system if the interference is not properly managed. Therefore, intelligent interference management needs to be considered in order to make the benefits of D2D communication available. Most of the related studies focused on mitigating the cross-tier interference from D2D pairs to CUs but cotier interference amongst D2D pairs is not tackled.

In this work, we design a network-assisted distributed interference coordination algorithm for D2D communication underlaid cellular networks to improve the data rate of D2D transmission while fulfilling the Quality of Service (QoS) requirements of CUs; we also consider the fairness amongst D2D pairs. The QoSs of CUs are ensured using a taxation scheme. Specifically, the BS maintains a reuse price which each D2D pair has to pay in order to reuse the radio resources. The interference from D2D transmission can be controlled by tuning this reuse price. Each D2D pair can reuse all radio resources of CUs and has to decide the transmit power on each resource; we modeled this process using noncooperative game theory [3]. To obtain a fair and efficient outcome, we introduce the reference D2D pair into the payoff function of each D2D pair. This is encouraged by the reference line approach in digital subscriber line [4]. Roughly speaking, the reference D2D pair is the victim of cotier interference and 
has the worst channel condition, by factoring the data rate of the reference D2D pair into the payoff function of each D2D pair as a taxation term, weak channel D2D pairs can be protected from excessive cotier interference. To characterize the Nash Equilibrium (NE) of the formulated game, we borrow the convenience of Variational Inequality (VI) theory [5]. Sufficient conditions for the existence and uniqueness of the NE are given based on VI theory. In addition, condition for the convergence of the proposed algorithm is also given. The proposed scheme is network-assisted distributed because it requires assistance from the BS to obtain information about the reference D2D pair. Finally, simulation results are given to evaluate the superiority of the proposed scheme.

In a nutshell, features and contributions offered by this work can be listed as follows:

(i) A system model that permits multiple D2D pairs to utilize the resource of multiple CUs is designed in Section 3. To overcome the selfishness of D2D pairs, we introduce the concept of the reference user and consider an efficient optimization problem at each D2D pair.

(ii) We also propose a network-assisted distributed approach for interference coordination in Section 4. The proposed scheme does not require information passing amongst D2D pairs since information about the reference D2D pair is provided by BS (we considered a network-assisted approach since the BS has the capability of providing information about the reference D2D pair) to address the problem of cotier interference amongst D2D pairs using the concept of game theory.

(iii) A Cellular Link Performance Management is also designed in Section 4 to address the cross-tier interference from D2D pairs to CUs and guarantee QoS of CUs.

(iv) Finally, through numerical simulation conducted in Section 6, it is shown that our proposed algorithm is close to the optimal scheme and is superior to existing frameworks, for example, the selfish version, the algorithm proposed in [6], and the scheme where all users (cellular users and D2D pairs) operate in the cellular mode.

\section{Related Works}

To date existing resource allocation schemes in this realm can be found in [6-24]. In $[7,8]$ the system throughput of a CU and a $\mathrm{D} 2 \mathrm{D}$ pair is maximized considering the target rate of CU. In [9], multiple CUs and a single D2D pair can coexist outside an interference restricted region. In [10], a centralized heuristic approach is proposed in which the resource of CU and D2D pair is matched based on the interference link gain from D2D transmitter to the BS; however the solution obtained can be far from optimal. In [11], with the aim of enhancing the system sum-rate of machine-type D2D links a joint mode selection and resource assignment scheme was designed by the author. The authors of [12] enhance the system capacity of multiple D2D pairs and CUs while fulfilling the QoSs of CUs and D2D pairs concurrently. However, the works in [7-12] are centralized which requires full knowledge of Link State Information (CSI), resulting in excessive information passing for most practical networks. In addition, the centralized optimization problem can be nonconvex and associates with complex computation that increases exponentially with the size of the input. In practical design, distributed resource allocations with less information passing and low computational complexity are more preferred. A distributed algorithm based on coalition game approach for multiple D2D pairs and CUs was studied in [13] under the assumption that D2D pairs and CUs can cooperate to improve the sum-rate. An incentive compatible pricing approach was adopted in [14] where the BS suppresses the interference from D2D transmission via pricing scheme. Nevertheless, this scheme can be improved significantly if the interference amongst D2D pairs is accounted for. An interference pricing scheme was design in [15] to manage interference from D2D communication; however, this work can be improved if interference amongst D2D pairs is managed. Distributed schemes in $[16,17]$ are proposed based on Stackelberg and auction games, respectively. However, in these works [7$10,12-17]$, at most one resource of CU can be reused by a D2D pair, which may not lead to high resource utilization.

Recently, some works have been done allowing each D2D pair to reuse multiple radio resources of CUs [6, 18-24]. In this scenario, the author in [18] used a merge-and-split technique to design an overlapping coalition game with the aim of maximizing the overall sum-rate. However, the performance is crippled by high signaling amongst D2D pairs. A joint problem of channel assignment and power allocation was considered in [19], where the D2D channel assignment is implemented centralizedly at the BS and the power allocation for D2D pairs is formulated as a Stackelberg game. Introducing the concept of the interference temperature constraint in D2D networks, the authors in [20] modeled the interaction between the D2D-tier and cellular tier as a Stackelberg game, where the BS sells interference to D2D pairs to maximize its utility while D2D pairs purchase interference from the BS and the competition amongst D2D pairs is formulated as a noncooperative power control game. The fairness amongst D2D pairs is however not considered in $[19,20]$. An efficient resource assignment scheme for multiple $\mathrm{D} 2 \mathrm{D}$ pairs with the objective of maximizing the total system capacity was studied in [21]. Nevertheless, interference amongst D2D pairs was not discussed. The authors in [22] show that the average data rate of the D2D communication can be significantly improved; however the channel assigned to a $\mathrm{CU}$ is allowed to be reused by only one $\mathrm{D} 2 \mathrm{D}$ pair and the cotier interference between D2D pairs is neglected. In [6], minimum data rates of CUs and D2D pairs are guaranteed using a distributed resource allocation scheme; however this method is valid only for suitable small data rate requirements of D2D pairs which may result in low spectral efficiency. In [23], the authors propose a distributed scheme using the convenience of noncooperative Stackelberg game which results in the reduction of signaling overhead with compromised system performance. In addition, the selfishness of each D2D pair in the formulated game may lead to inefficient outcome. In [24], 
TABLE 1: Comparison of the proposed mechanism with existing proposals.

\begin{tabular}{lcccc}
\hline Proposal & Distributed algorithm & Cross-tier interference & Cotier interference & Fairness \\
\hline$[6]$ & Yes & Yes & No & No \\
{$[7-12]$} & No & Yes & No & No \\
{$[13-18]$} & Yes & Yes & Yes & No \\
{$[19]$} & Yes & Yes & Yes & No \\
{$[20]$} & Yes & Yes & No \\
{$[21]$} & No & Yes & No \\
{$[22,23]$} & Yes & Yes & Yes & No \\
{$[24]$} & Yes & Yes & Yes & Yes \\
Proposed algorithm & Yes & Yes & & \\
\hline
\end{tabular}

a distributed resource allocation algorithm to enhance the sum data rate of $\mathrm{D} 2 \mathrm{D}$ pairs is designed; however this scheme is only optimal with a high Signal-to-Interference-plus-Noise Ratio (SINR) approximation; also, the fairness amongst D2D pairs is not considered.

Based on analysis of related literature, comparison between our considered problem and existing studies can be summarized in Table 1. From this, our work is the first to investigate a distributed algorithm of spectrum and power allocation in D2D communication with consideration of cross-tier interference, cotier interference, and fairness. Moreover, in our work, a CU can use multiple subchannels and a subchannel occupied by a $\mathrm{CU}$ is allowed to be reused by multiple D2D pairs.

\section{System Model and Problem Formulation}

In this work, we consider a single cell Orthogonal Frequency Division Multiple Access (OFDMA) which consists of $N$ subchannels and each D2D pair can reuse all the subchannels for communication. The system is made up of two tiers: the cellular tier and D2D tier; $\mathscr{D}=\{1, \ldots, D\}$ and $\mathscr{C}=$ $\{1, \ldots, C\}$ are the sets of D2D pairs and CUs, respectively. All transmitters and receivers are equipped with one antenna. In the cellular tier, both spectrum assignment and power control decisions for CUs are handled by the base station. The subchannels are orthogonally assigned to the CUs; hence there is no cotier interference amongst CUs. In D2D tier, the competition for reusing the resources is handled in a decentralized fashion using noncooperative game. Due to the overlapping of the resources of D2D pairs, cotier interference amongst D2D pairs exists and should be mitigated. Additionally, D2D pairs coexist on the same subchannels with CUs so cross-tier interference between CUs and D2D pairs do exist and should also be mitigated.

We focus on uplink transmission since, in this case, the victim of cross-tier interference is the BS, which has the capability of handling the interference more efficiently than mobile users, for example, using smart antenna techniques [25]. We assume that the channel conditions on all subchannels are fixed during the time of interest (e.g., in slow mobility scenario). During the uplink phase the BS is subjected to cross-tier interference from D2D pairs transmitting on the same subchannel with a $\mathrm{CU}$. Let $\mathrm{CU}_{c}$ be $\mathrm{CU} c$ and $\mathrm{D}_{2} \mathrm{D}_{d}$ be

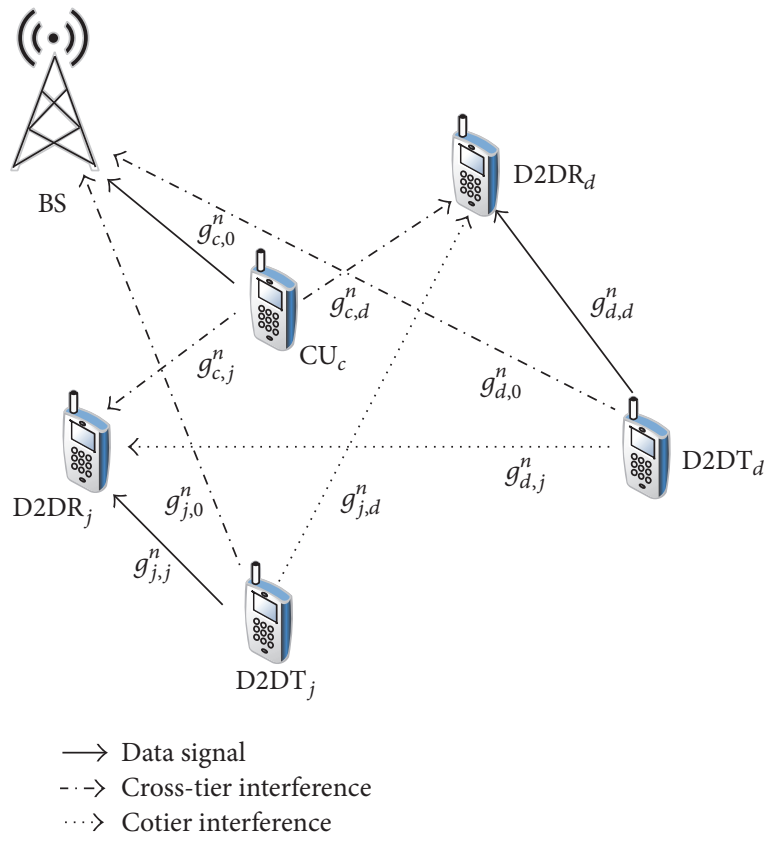

FIGURE 1: Cotier and cross-tier interference in D2D transmission reusing the uplink cellular resources.

$\mathrm{D} 2 \mathrm{D}$ pair $d ; \mathrm{D}_{2} \mathrm{DT} T_{d}$ and $\mathrm{D} 2 \mathrm{DR}_{d}$ are the transmitter and receiver of $\mathrm{D}_{2} \mathrm{D}_{d}$, respectively. The SINR of $\mathrm{CU}_{c}$ on subchannel $n$ is formulated as

$$
\operatorname{SINR}_{c}^{n}=\frac{p_{c}^{n} g_{c, o}^{n}}{\mathrm{I}_{c}^{n}+\sigma^{2}},
$$

where $\mathrm{I}_{c}^{n}=\sum_{d \in \mathscr{D}} p_{d}^{n} g_{d, o}^{n}$ represents the total interference from D2DTs to $\mathrm{CU}_{c}$ and $g_{d, o}^{n}$ and $g_{c, o}^{n}$ are the link gains from $\mathrm{D} 2 \mathrm{DT}_{d}$ and $\mathrm{CU}_{c}$ to the $\mathrm{BS}$, respectively (Figure 1). $p_{c}^{n}$ and $p_{d}^{n}$ are the transmit powers of $\mathrm{CU}_{c}$ and $\mathrm{D}_{2} \mathrm{D}_{d}$ on subchannel $n$, respectively and $\sigma^{2}$ is the noise variance. $\mathrm{D} 2 \mathrm{DR}_{d}$ on subchannel $n$ also suffers interference from $\mathrm{CU}_{c}$ on subchannel $n$ and other $\mathrm{D}_{2} \mathrm{D}_{j}$ sharing the same subchannel. The SINR of $\mathrm{D}_{2} \mathrm{D}_{d}$ pair on subchannel $n$ is formulated as

$$
\operatorname{SINR}_{d}^{n}=\frac{p_{d}^{n} g_{d, d}^{n}}{p_{c}^{n} g_{c, d}^{n}+\mathrm{I}_{d}^{n}+\sigma^{2}},
$$


where $\mathrm{I}_{d}^{n}=\sum_{j \in \mathscr{D}, j \neq d} p_{j}^{n} g_{j, d}^{n}$ represents interference from neighbouring D2D pairs; $g_{j, d}^{n}$ and $g_{c, d}^{n}$ are the link gains between $\mathrm{D}^{2 \mathrm{DT}}{ }_{j}$ to $\mathrm{D}_{2} \mathrm{DR}_{d}$ and $\mathrm{CU}_{c}$ to $\mathrm{D}_{2} \mathrm{DR}_{d}$ on subchannel $n$, respectively. $p_{d}^{n}$ and $p_{j}^{n}$ are the transmit powers of $\mathrm{D}_{2} \mathrm{DT}_{d}$ and $\mathrm{D} 2 \mathrm{DT}_{j}$ on subchannel $n$, respectively. The achievable data rates of $\mathrm{CU}_{c}$ and $\mathrm{D}_{2} \mathrm{D}_{d}$ on subchannel $n$ are given, respectively, as

$$
\begin{aligned}
R_{c} & =\sum_{n=1}^{N} r_{c}^{n}, \\
R_{d} & =\sum_{n=1}^{N} r_{d}^{n},
\end{aligned}
$$

where $r_{c}^{n}=\log _{2}\left(1+\operatorname{SINR}_{c}^{n}\right)$ and $r_{d}^{n}=\log _{2}\left(1+\operatorname{SINR}_{d}^{n}\right)$ are the data rates of $\mathrm{CU}_{c}$ and $\mathrm{D} 2 \mathrm{D}_{d}$ on subchannel $n$, respectively and $\mathcal{N}(c)$ is the subchannel set assigned to $\mathrm{CU}_{c}$.

The main aim of this paper is to develop a fairness-aware distributed interference management scheme for D2D communications underlaid cellular networks while guaranteeing the QoS requirement of CUs. To fulfil these tasks, a taxation scheme is developed at BS to manage the interference resulting from D2D communication while the transmit power of D2DTs are modeled as a noncooperative power control game. Specifically, the BS charges each D2D $\mathrm{D}_{d}$ a price, $\boldsymbol{\theta}=\left(\theta^{n}\right)_{n=1}^{N}$, for reusing the subchannel $n$. When the $\mathrm{D} 2 \mathrm{D}$ pairs receive the price on each subchannel, they compete for the reuse of the subchannels in a noncooperative game fashion; we now introduce the game formulation as follows.

Generally, game theory is used to analyze complex decision problems amongst decision makers. One can consider the simplest game model $\mathscr{G}$ given by $\mathscr{G}=\left\{\mathscr{D},\left(\mathscr{P}_{d}\right)_{d \in D}\right.$, $\left.\left(u_{d}\right)_{d \in D}\right\}$, where the set of players is $\mathscr{D} ; u_{d}$ is the reward (the achievable data rate minus the cost paid to the $\mathrm{BS}$ ) $\mathrm{D}_{2} \mathrm{D}_{d}$ pair receives. Each D2D pair selfishly solves the optimization problem below as follows:

$$
\max _{\mathbf{p}_{d} \in \mathscr{P}_{d}} u_{d}=R_{d}-\sum_{n=1}^{N} \theta^{n} p_{d}^{n} g_{d, o}^{n},
$$

where the feasible set of strategies is

$$
\begin{aligned}
\mathscr{P}_{d} & =\left\{\mathbf{p}_{d}=\left[\left(p_{d}^{n}\right)_{n=1}^{N}\right]: \sum_{n=1}^{N} p_{d}^{n} \leq P_{d, \max }, 0 \leq p_{d}^{n}\right. \\
& \left.\leq p_{d, \max }^{n}\right\},
\end{aligned}
$$

and $P_{d, \max }$ and $p_{d, \max }^{n}$ are the power budget and maximum transmit power on subchannel $n$, respectively. In the above game formulation, there is no need for exchange of information amongst D2D pairs. However, the D2D pairs performance is sacrificed owing to the rational behaviour of the D2D pairs. To obtain a fair and efficient outcome, we introduced the idea of reference D2D pair which represents the typical victim of cotier interference on each subchannel $n$ [4]. Specifically, the data rate of the reference D2D pair is factored into the reward which each $\mathrm{D} 2 \mathrm{D}_{d}$ can receive to mitigate the cotier interference cause to other $\mathrm{D} 2 \mathrm{D}$ pairs as a result of the selfishness of $\mathrm{D} 2 \mathrm{D}_{d}$. The reference $\mathrm{D} 2 \mathrm{D}$ pair is chosen such that

$$
\operatorname{ref}_{d}^{n}=\arg \min _{d \in \mathscr{D}} g_{d, d}^{n}
$$

From each D2D pairs perspective, the data rate of the reference $\mathrm{D} 2 \mathrm{D}$ pair is given as

$$
R_{d, \text { ref }}=\sum_{n=1}^{N} \log _{2}\left(1+\frac{p_{\text {ref }_{d}^{n}}^{n} g_{\text {ref }_{d}^{n}}^{n}}{p_{c}^{n} g_{c, \text { ref }}^{n}+p_{d}^{n} g_{d, \text { ref }}^{n}+\sigma^{2}}\right) .
$$

With the concept of reference D2D pair, unless otherwise specified, we consider the game $\mathscr{G}=\left\{\mathscr{D},\left(\mathscr{P}_{d}\right)_{d \in D},\left(U_{d}\right)_{d \in D}\right\}$ with the payoff function $U_{d}$ is given by

$$
U_{d}=R_{d}+R_{d, \mathrm{ref}}-\sum_{n=1}^{N} \theta^{n} p_{d}^{n} g_{d, o}^{n}
$$

Roughly speaking, $R_{d \text {,ref }}$ can be interpreted as a taxation term in each D2D pair optimization problem to adjust its rational behaviour to a more social behaviour. Now each D2D pair aims at solving the maximization problem below.

$$
\max _{\mathbf{p}_{d} \in \mathscr{P}_{d}} U_{d}
$$

\section{D2D Resource Allocation and Cellular Interference Management}

In this section, we show the solution of the game $\mathscr{G}$ and the cellular interference management.

4.1. D2D Resource Allocation. First notice that problem (9) is nonconvex owing to the inclusion of the reference D2D pair in the payoff function, fortunately based on the asymptotic result in [4] that the duality gap approaches zero as the number of subchannels increases to infinity. Exploiting this fact, the best response (the optimal solution of (9)) for each

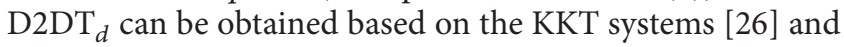
is given in water-filling form as follows:

$$
\begin{aligned}
p_{d}^{n} & =\mathrm{WF}\left(\mathbf{p}_{-d} ; \boldsymbol{\theta}\right)_{n=1}^{N} \triangleq\left[\frac{1}{\left(\lambda_{d}+\theta^{n} g_{d, o}^{n}\right) \ln 2+t_{d}^{n}}\right. \\
& \left.-\frac{p_{c}^{n} g_{c, d}^{n}+\mathrm{I}_{d}^{n}+\sigma^{2}}{g_{d, d}^{n}}\right]_{0}^{p_{d, \max }^{n}}, \quad \forall n \in\{1, \ldots, N\},
\end{aligned}
$$

where

$$
\begin{aligned}
& t_{d}^{n}=\frac{g_{d, \mathrm{ref}_{d}^{n}}^{n} \operatorname{SINR}_{\mathrm{ref}_{d}^{n}}^{n}}{\left(p_{c}^{n} g_{c, \mathrm{ref}_{d}^{n}}^{n}+p_{d}^{n} g_{d, \mathrm{ref}_{d}^{n}}^{n}+\sigma^{2}+p_{\mathrm{ref}_{d}^{n}}^{n} g_{\text {ref }_{d}^{n}}^{n}\right) \ln 2}, \\
& \forall d \in \mathscr{D}, n \in N,
\end{aligned}
$$

and $[b]_{x}^{y}=\min \{y,\{\max \{b, x\}\}\} ; \mathbf{t}_{d}=\left(t_{d}^{n}\right)_{n=1}^{N}$ represents the effect interference $\mathrm{D} 2 \mathrm{D}_{d}$ causes to the reference $\mathrm{D} 2 \mathrm{D}$ pair; 
(1) Initialize parameters $\mathbf{p}_{d} \in \mathscr{P}_{d}, \boldsymbol{\theta}=0$, and $t_{d}^{n} \geq 0$

(2) Reference D2D pair selection according to (6)

(3) Set the counter $\tau=0$ and $L^{\max }$

(4) while $\tau<L^{\max }$ do

(5) for $n \in N, d \in \mathscr{D}$ do

(6) Update $p_{d}^{n}(\tau+1)$ according to (10)

(7) Update $t_{d}^{n}(\tau+1)$ according (11)

(8) end for

(9) end while

Algorithm 1: Fair Iterative Water-filling Algorithm (FIWA).

$\mathbf{p}_{-d}$ is the power vector of all D2Ds except $\mathrm{D}_{2} \mathrm{D}_{d}$. The dual variable $\lambda_{d}$ is associated with the constraint $\sum_{n=1}^{N} p_{d}^{n} \leq P_{d}$. The optimal dual variables $\lambda_{d}^{*}$ can be obtained such that $\sum_{n=1}^{N} p_{d}^{n}=P_{d}$. The proposed resource allocation algorithm for D2D pairs is summarized in Algorithm 1.

Remark 1. To determine the reference D2D pair, first each $\mathrm{D} 2 \mathrm{D}$ pair reports its own link gain to the BS to choose the reference pair based on (6). After this, $\operatorname{ref}_{d}^{n}$ has to reports the following additional information to the BS:

(i) The interference link gain from other pairs (i.e., $\left.g_{d, \mathrm{ref}_{d}^{n}}^{n}\right)$ in (11).

(ii) The $\operatorname{SINR}_{\text {ref }_{d}^{n}}^{n}$ in (11).

Also the $\mathrm{D}_{2} \mathrm{DT}_{d}$ needs to reports its transmit power to the $\mathrm{BS}$; then the $\mathrm{BS}$ is able to derive the interference plus noise (i.e., $p_{c}^{n} g_{c, \text { ref }}^{n}+p_{d}^{n} g_{d, \text { ref }}^{n}+\sigma^{2}$ ) in (11) and broadcasts all these required information on its control channel to all D2D pairs to update their transmit powers. Note that in this procedure, the burden of collecting and disseminating the information is shifted at the BS side.

Remark 2. The main difference between Fair Iterative Waterfilling Algorithm (FIWA) and Iterative Water-filling Algorithm (IWA) in [27] is the incorporation of the reference D2D pair taxation term $t_{d}^{n}$ which controls the effect of interference to reference D2D pair. When reference D2D pair taxation term is not considered, $t_{d}^{n}=0$, FIWA is reduced to IWA. By including $t_{d}^{n}>0$ each D2D pair acts in a more social manner taking into consideration the interference to the reference D2D pair. The complexity of the FIWA for a given reuse price $\boldsymbol{\theta}$ is $\mathcal{O}\left(N \log _{2} N+N D\right)$ since obtaining the solution of the water-filling system in (10) requires a computational burden $\mathcal{O}\left(N \log _{2} N\right)$ and selecting the reference D2D pair on each subchannel in (6) has a complexity $\mathcal{O}(N D)$. The convergence properties of FIWA are characterized in Section 5.

4.2. Cellular Link Performance Management. The CUs being the primary users in the network need to be protected from cross-tier interference resulting from D2D users communicating on the same channel as the CUs. The QoS of $\mathrm{CU}_{c}$ is given as

$$
R_{c} \geq r_{c}^{\min }, \quad \forall c \in \mathscr{C}
$$

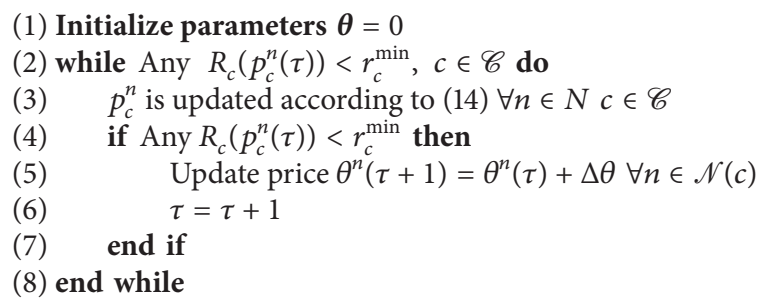

Algorithm 2: Cellular Link Performance Management (CLPM).

where $r_{c}^{\min }$ is the rate requirement of $\mathrm{CU}_{c}$. Now to perform this task, we first update the transmit power of each $\mathrm{CU}_{c}, \forall c \in \mathscr{C}$, first; if it fails to guarantee (12) then the price on the corresponding subchannels in $\mathscr{N}(c)$ will be adjusted until the QoS is met; that is, each $\mathrm{CU}_{c}$ first solves the following optimization problem:

$$
\begin{aligned}
\max _{0 \leq \mathbf{p}_{c} \leq \mathbf{p}_{c}^{\max }} & R_{c} \\
\text { s.t. } & \sum_{n \in \mathcal{N}(c)} p_{c}^{n} \leq P_{c, \max },
\end{aligned}
$$

where $\mathbf{p}_{c}=\left[\left(p_{c}^{n}\right)_{n \in \mathcal{N}(c)}\right], \mathbf{p}_{c}^{\max }=\left[\left(p_{c, \max }^{n}\right)_{n \in \mathbb{N}(c)}\right]^{T}$, and $p_{c, \max }^{n}$ is the highest transmit power allowed on subchannel $n ; P_{c, \max }$ is the total transmit power budget. Since each subchannel is exclusively assigned to one $\mathrm{CU}$ at the cellular tier, optimization problem (13) is convex; the solution is easily obtained using KKT conditions and is given in water-filling form as

$$
p_{c}^{n}=\left[\frac{1}{\ln 2 \beta}-\frac{\mathrm{I}_{c}^{n}+\sigma^{2}}{g_{c, o}^{n}}\right]_{0}^{p_{c, \max }^{n}},
$$

where $\beta$ is the Lagrange multiplier chosen to meet $\sum_{n \in \mathcal{N}(c)} p_{c}^{n}=P_{c, \max }$. At this point, if $R_{c} \geq r_{c}^{\min }$, then the cross-tier interference price will not be adjusted, otherwise the BS will adjust the price by

$$
\theta^{n}(\tau+1)=\theta^{n}(\tau)+\Delta \theta, \quad \forall n \in \mathcal{N}(c),
$$

where $\Delta \theta$ is a positive step size. The Cellular Link Performance Management (CLPM) is summarized in Algorithm 2.

\section{Convergence Analysis}

We now characterize the convergence of the FIWA. We first recall the definition of Nash Equilibrium (NE) [3].

Definition 3. A power profile $\left(\mathbf{p}_{d}^{*}, \mathbf{p}_{-d}^{*}\right)$ is a NE of the game $\mathscr{G}$ if it is a fixed point of the best response for each $\mathrm{D}_{2} \mathrm{D}_{d}$ in (10); that is,

$$
U_{d}\left(\mathbf{p}_{d}^{*}, \mathbf{p}_{-d}^{*}\right) \geq U_{d}\left(\mathbf{p}_{d}^{\prime}, \mathbf{p}_{-d}^{*}\right), \quad \forall \mathbf{p}_{d}^{\prime} \in \mathscr{P}_{d}, \forall d \in \mathscr{D} .
$$

Intuitively, this means that at the NE, no D2D pair can obtain higher payoff by unilaterally adjusting its transmit power given the transmit powers of other D2DTs remain 
fixed. In general, it is hard to analyze the existence and uniqueness of the NE of $\mathscr{G}$ since the fixed point of the water-filling system in (10) is not easy to characterize. So we turn to Variational Inequality (VI) theory [5], a powerful mathematical tool which is able to address these problems. Let us recall the definition of VI problem as follows.

Definition 4. Given a closed and convex set $\mathscr{K} \subseteq \Re^{n}$ and a mapping $\mathbf{F}: \mathscr{K} \rightarrow \mathfrak{R}^{n}$, the VI problem $\mathrm{VI}(\mathscr{K}, \mathbf{F})$ is to find a vector $x^{*} \in \mathscr{K}$ (called the solution of the VI) such that [5]

$$
\left(\mathbf{y}-\mathbf{x}^{*}\right)^{T} \mathbf{F}\left(\mathbf{x}^{*}\right) \geq 0 \quad \forall y \in \mathscr{K} .
$$

The similarity between the game $\mathscr{G}$ and VI problem is given in the following proposition.

Proposition 5. The NE of $\mathscr{G}$ is equivalent to the solution of $\mathrm{VI}(\widehat{\mathscr{P}}, \mathbf{F})$, where $\widehat{\mathscr{P}} \triangleq \prod_{d}^{D} \mathscr{P}_{d}$ and the mapping $\mathbf{F}(\mathbf{P})=$ $\left[\left(\mathbf{F}_{d}(\mathbf{P})\right)_{d \in \mathscr{D}}\right]^{T}$ is given by

$$
\mathbf{F}_{d}(\mathbf{P})=-\nabla U_{d}(\mathbf{P})
$$

where $\mathbf{P}=\left(\mathbf{p}_{d}\right)_{d=1}^{D}$.

Proof. If $\mathbf{P}^{*}$ is the NE of $\mathscr{G}$, then $\mathbf{p}_{d}^{*}, \forall d \in \mathscr{D}$ is the optimal solution of the optimization problem (9) and it has to satisfy the maximum principle $\left(\mathbf{p}_{d}-\mathbf{p}_{d}^{*}\right)^{T} \mathbf{F}_{d}(\mathbf{P}) \geq 0, \forall \mathbf{p}_{d} \in \mathscr{P}_{d}$. Summing this condition over all $d \in \mathscr{D}$ and considering the Cartesian product of $\widehat{\mathscr{P}}$ leads to a connection between the NE of $\mathscr{G}$ and the solution of $\operatorname{VI}(\widehat{\mathscr{P}}, \mathbf{F})$.

We now proceed to the introduction of the main theorems.

Theorem 6. Given $\boldsymbol{\theta} \geq 0$ and $\mathbf{t}_{d}$ is fixed, $\mathscr{G}$ always admits $N E$.

Proof. From the result of Proposition 5, the NE of $\mathscr{G}$ is similar to the solution of $\operatorname{VI}(\widehat{\mathscr{P}}, \mathbf{F})$; it all remains to show that $\mathrm{VI}(\widehat{\mathscr{P}}, \mathbf{F})$ admits at least one solution. Based on the result in [5], $\operatorname{VI}(\widehat{\mathscr{P}}, \mathbf{F})$ has at least one solution if $\mathbf{F}$ is continuous and $\widehat{\mathscr{P}}$ is compact and convex. From definitions (5) and the payoff functions in (8), (17), and (18), it is easy to show that $\mathbf{F}$ is continuous and $\widehat{\mathscr{P}}$ is compact and convex; this completes the proof.

Theorem 7. Given $\boldsymbol{\theta} \geq 0$ and $\mathbf{t}_{d}$ fixed, $\mathscr{G}$ admits a unique $N E$ if $\mathbf{A}$ is positive definite (PD) where $\mathbf{A}$ is defined as

$$
[\mathbf{A}]_{d, j}= \begin{cases}1, & \text { if } j=d, \\ -\max _{1 \leq n \leq N}\left\{\bar{g}_{j, d}^{n} \zeta_{j, d}^{n}\right\}, & \text { if } j \neq d,\end{cases}
$$

where $\zeta_{j, d}^{n}$ is given as

$$
\zeta_{j, d}^{n}=\frac{p_{j, \max }^{n}+\left(\sum_{j=1, r \neq j}^{D} p_{r, \max }^{n} \bar{g}_{r, d}^{n}+\bar{\sigma}_{j}^{n}\right)}{\bar{\sigma}_{d}^{n}},
$$

where $\bar{\sigma}_{d}^{n} \triangleq\left(p_{c}^{n} g_{c, d}^{n}+\sigma^{2}\right) / g_{d, d}^{n}$ and $\bar{g}_{r, d}^{n} \triangleq g_{r, d}^{n} / g_{d, d}^{n}$.

Proof. See Appendix.
Theorem 8. The convergence of the FIWA is guaranteed when

$$
\rho\left(\mathbf{H}_{\max }\right)<1,
$$

where $\rho\left(\mathbf{H}_{\max }\right)$ is the spectral radius of $\mathbf{H}_{\max }$ and $\mathbf{H}_{\max }$ is a $D \times D$ matrix defined as

$$
\left[\mathbf{H}_{\max }\right]_{d, j}= \begin{cases}\max _{1 \leq n \leq N} \bar{g}_{j, d}^{n} \frac{p_{j, \max }^{n},}{p_{d, \max }^{n},} & \text { if } j \neq d, \\ 0, & \text { otherwise, }\end{cases}
$$

where $\bar{g}_{j, d}^{n} \triangleq g_{j, d}^{n} / g_{d, d}^{n}$

Proof. The solution of a $\operatorname{VI}(\widehat{\mathscr{P}}, \mathbf{F})$ can be seen as Euclidean projection of a proper map onto the convex set $\mathbf{P}$ [5]. Therefore, based on the fact that the projection in (10) is nonexpansion [5] and the condition $\rho\left(\mathbf{H}^{\max }\right) \leq 1$ we have for each $d \in \mathscr{D}$

$$
\begin{aligned}
& \left\|\mathrm{WF}\left(\mathbf{p}_{-d}(t), \boldsymbol{\theta}\right)-\mathrm{WF}\left(\mathbf{p}_{-d}(t+1), \boldsymbol{\theta}\right)\right\| \\
& \quad \leq\left\|\mathbf{H}_{\max }\left(\mathbf{p}_{d}(t)-\mathbf{p}_{d}(t+1)\right)\right\| \\
& \leq\left\|\mathbf{H}_{\max }\right\|\left\|\mathbf{p}_{d}(t)-\mathbf{p}_{d}(t+1)\right\| \\
& \leq\left\|\mathbf{p}_{d}(t)-\mathbf{p}_{d}(t+1)\right\| .
\end{aligned}
$$

This means that the water-filling mapping in (10) is a contraction one, following the Banach Contraction Theorem; the convergence of the FIWA is verified.

Corollary 9. The sufficient condition (19) of Theorem 7 for the game $\mathscr{G}$ to admit a unique $N E$ is more binding than the convergence condition (21) of Theorem 8.

Proof. From Theorem 7, $\mathscr{G}$ admits a unique NE when the matrix $\mathbf{A}$ is $\mathrm{PD}$; since $\mathrm{PD}$ matrix is also $P$-matrix, we have $\rho(\mathbf{I}-\mathbf{A})<1$ [27] where I denote a unit matrix. Therefore $\lim _{l \rightarrow \infty}(\mathbf{I}-\mathbf{A})^{l}=0[28]$; since $\zeta_{d}^{n}>p_{j, \max }^{n} / p_{d, \text { max }}^{n}$, $\lim _{l \rightarrow \infty}\left(\mathbf{H}_{\max }\right)^{l}=0$. Because $\rho\left(\mathbf{H}_{\max }\right)<1$ if and only if $\lim _{l \rightarrow \infty}\left(\mathbf{H}_{\text {max }}\right)^{l}=0$ [27], we end the proof by concluding that condition (19) in Theorem 7 is more binding compared to the condition (21) in Theorem 8.

Corollary 9 means that, provided that the condition in (19) of Theorem 7 is met, the uniqueness of the NE is fulfilled and the FIWA globally converges to that NE. The sufficient condition for Theorems 7 and 8 can be made explicit as follows, based on [29] and [30, Theorem 5.6.9] we have

$$
\rho\left(\mathbf{H}_{\max }\right)=\rho\left((\mathbf{I}-\mathbf{A})^{T}\right) \leq\|\mathbf{I}-\mathbf{A}\|,
$$

where $\|\cdot\|$ is some matrix norms [30]. Hence, a sufficient condition for (21) is $\left\|\mathbf{H}_{\max }\right\|_{\infty}^{\mu} \leq 1$, where $\|\cdot\|_{\infty}^{\mu}$ represents the weighted block maximum norm, given as [30]

$$
\left\|\mathbf{H}_{\max }\right\|_{\infty}^{\mu} \triangleq \max _{d \in \mathscr{D}} \frac{1}{\mu_{d}} \sum_{j \neq d} \mu_{j}[\mathbf{I}-\mathbf{A}]_{d, j},
$$

where $\boldsymbol{\mu} \triangleq\left[\mu_{1}, \ldots, \mu_{D}\right]^{T}>0$. 
Based on (24) and (25), the necessary conditions for Theorem 7 are given as

$$
\begin{aligned}
& \max _{d} \frac{1}{\mu_{d}} \sum_{j \neq d} \mu_{j} \max _{1 \leq n \leq N}\left\{\bar{g}_{j, d}^{n} \zeta_{j, d}^{n}\right\}<1, \\
& \max _{j} \frac{1}{\mu_{j}} \sum_{d \neq j} \mu_{d} \max _{1 \leq n \leq N}\left\{\bar{g}_{j, d}^{n} \zeta_{j, d}^{n}\right\}<1 .
\end{aligned}
$$

Also, the conditions in Theorem 8 are

$$
\begin{aligned}
& \max _{d} \frac{1}{\mu_{d}} \sum_{j \neq d} \mu_{j} \max _{1 \leq n \leq N}\left\{\bar{g}_{j, d}^{n} \frac{p_{j, \text { max }}^{n}}{p_{d, \text { max }}^{n}}\right\}<1, \\
& \max _{j} \frac{1}{\mu_{j}} \sum_{d \neq j} \mu_{d} \max _{1 \leq n \leq N}\left\{\bar{g}_{j, d}^{n} \frac{p_{j, \max }^{n}}{p_{d, \text { max }}^{n}}\right\}<1 .
\end{aligned}
$$

Remark 10. From (26) and (27), the convergence of FIWA and uniqueness of NE in $\mathscr{G}$ are guaranteed if the cotier interference is not too high (note that this condition may not be satisfied in practice when there are too many close by D2D pairs that transmit at the same time; in such case, admission control is required to satisfy this condition, e.g., setting a high target SINR in the discovery phase of a D2D pair). The achievement of NE is independent of the crosstier interference price vector and cross-tier interference. The above condition can be considered as admission control for a pair to communicate in $\mathrm{D} 2 \mathrm{D}$ mode.

\section{Simulation Results}

In this section, we conduct simulations to access the performance of the CLPM and FIWA. A single cell scenario is considered with radius of $500 \mathrm{~m}$, and the BS is situated at the centre of the cell. CUs and D2Ds are randomly located in the cell at a distance of at least $1 \mathrm{~m}$ away from the BS to avoid the case where the distance between BS and mobile users is zero. In order for Theorems 7 and 8 to be met, the minimum separation amongst any two different D2Ds $\left(\mathrm{D}_{2} \mathrm{D}_{d}\right.$ and $\mathrm{D}_{2} \mathrm{D}_{j}$ ) is set to at least $50 \mathrm{~m}$. An snapshot of the topology is given in Figure 2.

There are total of 16 subchannels, and each CU is allocated a set of subchannels. The subchannels are allocated to CUs in a Round Robin manner; that is, in the first round, one subchannel is allocated to the $\mathrm{CU}$ that has the best channel condition; then this $\mathrm{CU}$ is excluded in that particular round and the next subchannel is assigned to the next $\mathrm{CU}$ with the best link gain. This process is repeated until all subchannels are allocated to all CUs. The path loss is modeled as $37+$ $30 \log _{10}(d)$, where $d$ (meters) represents the transmitterreceiver distance [31]. The shadowing is modeled as lognormal random number with zero mean and standard deviation of $4 \mathrm{~dB}$. The noise variance is assumed as $-120 \mathrm{dBm}$ [31]. $P_{c, \text { max }}$ and $P_{d, \max }$ are normalized by the maximum transmit power as $1, \forall c \in \mathscr{C}$ and $\forall d \in \mathscr{D}$, respectively. The rate requirement of the $\mathrm{CU}_{c}$ is $r_{c}^{\mathrm{min}}=8.37 \mathrm{bits} / \mathrm{s} / \mathrm{Hz}, \forall c \in \mathscr{C}$. Finally, we set $\Delta \theta=3.5 \times 10^{5}$.

We first investigate the convergence of the proposed scheme. Figures 3 and 4 demonstrate the convergences of the

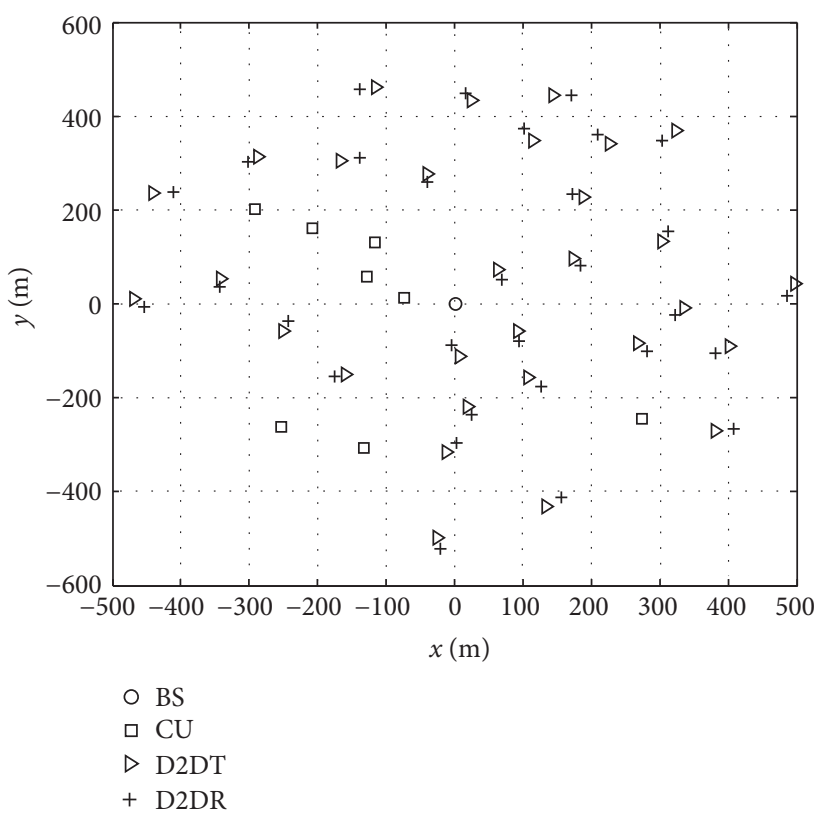

FIGURE 2: An example of topology.
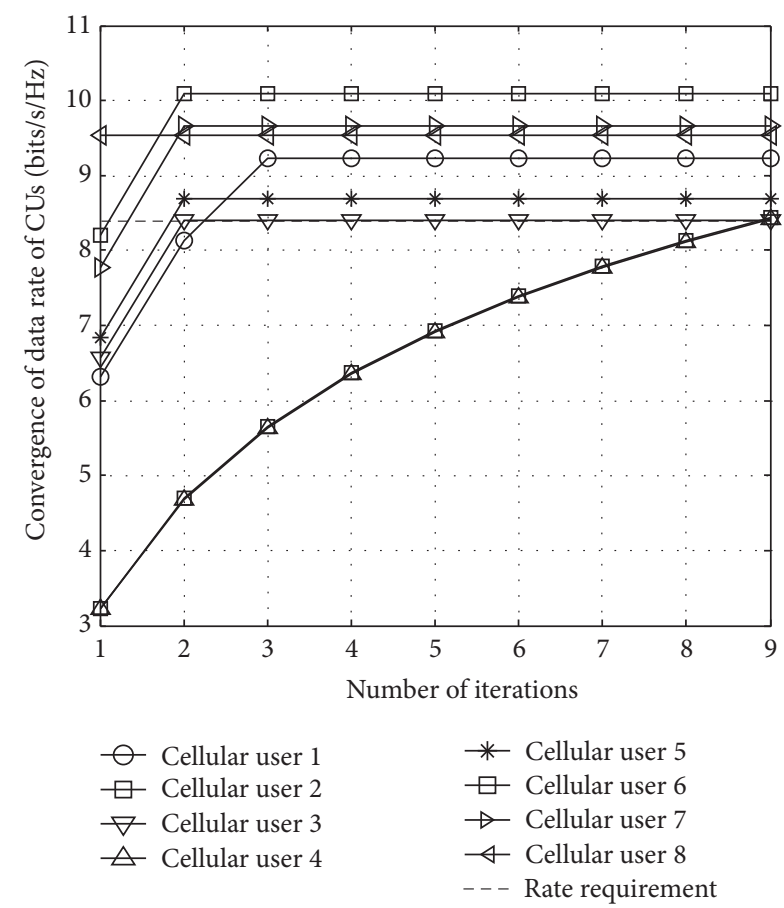

FIgURE 3: Convergence of the data rate of each CU; $D=30$.

CLPM and FIWA, respectively, for one channel realization. It is observed in Figure 3 that during the initial iteration the CUs obtained a low data rate because D2D pairs transmit at high power (note that at this point the reuse price $\boldsymbol{\theta}=0$ ) causing severe interference to the cellular link. The BS senses the interference and updates the price on the corresponding subchannel forcing D2DTs to reduce their transmit powers; this results in the increment of the data rate of $\mathrm{CU}$ until it satisfies the target requirement. In Figure 4, we plot the data 


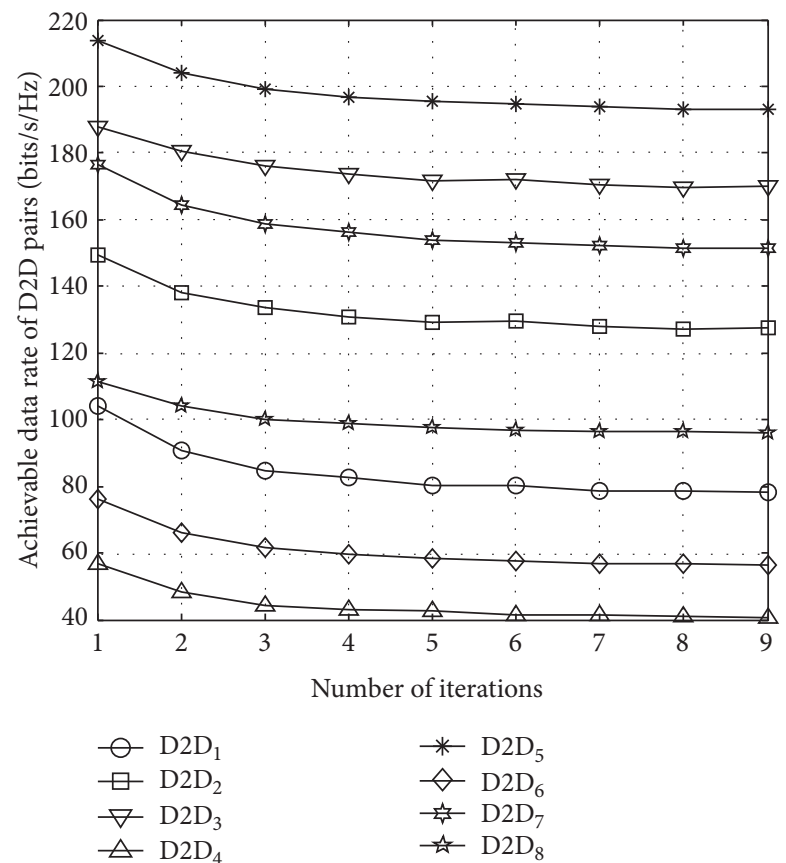

FIgURE 4: Convergence of the data rates of D2Ds; $C=8, D=8$.

rates of $\mathrm{D} 2 \mathrm{D}$ pairs during the noncooperative competition; it can be observed that the FIWA converges within 9 iterations.

In Figure 5 we compare the average data rate of D2D pair from the CLPM and FIWA with some existing algorithms. Note that the optimal solution is centralized and achieves maximum total reward $u_{d}$ of all $\mathrm{D}_{2} \mathrm{D}_{d}$; the optimal solution can be obtained by using the transformation $x_{d}^{n}=$ $\ln p_{d}^{n}$ as in [32]. The selfish version is the scheme in [23] where each $\mathrm{D} 2 \mathrm{D}_{d}$ rationally maximizes its own reward $u_{d}$ given in (4). For the scheme in [6], each D2DT selfishly minimizes the cost for reusing the resources while fulfilling its data rate requirement. This suggests that our proposed algorithm obtains the best gain because it manages the cotier interference amongst D2D pairs. Figure 5 demonstrates that our proposed algorithm significantly outperform the selfish version, the scheme in [6], and the cellular mode. Note that, in Figure 5, for any D2D pair to operate in the cellular mode, when $C+D \geq N$, more subchannels are added to support D2D pairs to communicate in cellular mode.

Lastly, in Figure 6 we demonstrate the fairness of the resource sharing based on Jain's fairness measure which is derived as [33]

$$
J_{(1, \ldots, D)}=\frac{\left(\sum_{d=1}^{D} R_{d}\right)^{2}}{D \cdot \sum_{d=1}^{D} R_{d}^{2}} ;
$$

this measurement determines how fair the resources are allocated to D2D pairs; note that $J_{(1, \ldots, D)}^{\max }=1$ when $R_{d}=R_{j}, \forall j \neq d, j, d \in \mathscr{D}$. As expected, cellular mode achieves the highest fairness since the subchannels are orthogonally assigned to CUs using a Round Robin scheme, resulting in roughly similar average data rates for all CUs.

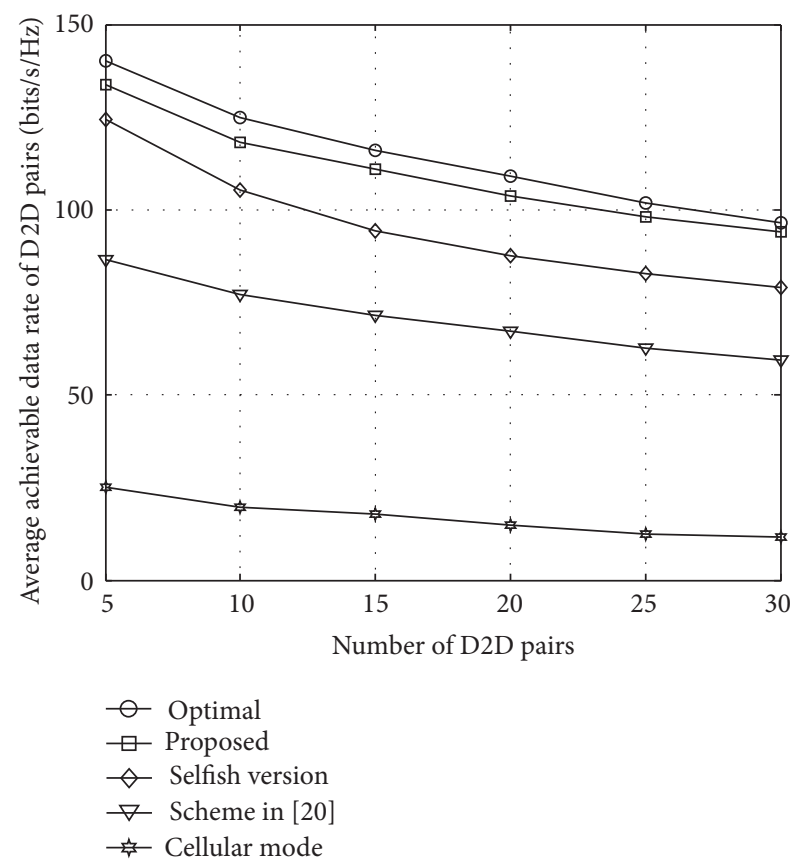

Figure 5: D2D data rates versus the number of D2D pairs; $C=8$.

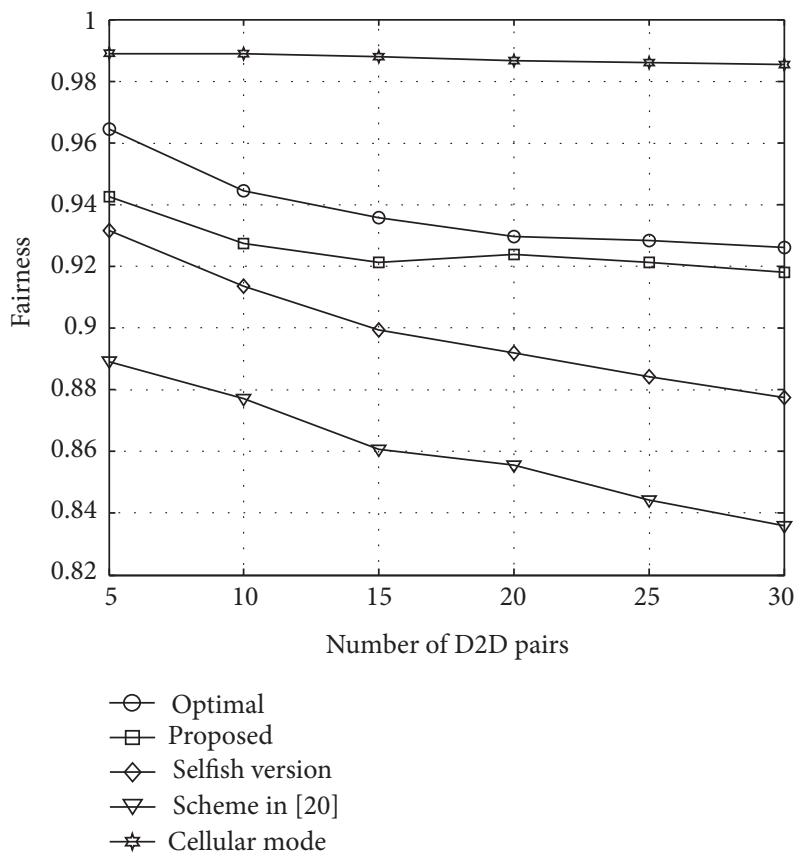

FIGURE 6: Fairness against the number of D2D pairs; $C=8$.

We observe that increasing number of $\mathrm{D} 2 \mathrm{D}$ pairs decreases the fairness measure of $\mathrm{D} 2 \mathrm{D}$ data transmission under the proposed scheme. The proposed algorithm obtains nearly same fairness measure as the optimal solution from the centralized algorithm. Both the selfish version [23] and the scheme in [6] achieved lower fairness measure due to selfish actions of $\mathrm{D} 2 \mathrm{D}$ pairs without considering the harm they cause to other $\mathrm{D} 2 \mathrm{D}$ pairs. 


\section{Conclusion}

In this work, we proposed a network-assisted distributed fairness-aware interference management scheme for D2D communications underlaid cellular networks to improve the data rate of D2D transmission while fulfilling the QoSs of CUs. In order to meet the QoSs of CUs, a taxation scheme was developed at the BS. The transmit power control problem amongst $\mathrm{D} 2 \mathrm{D}$ pairs is modeled using noncooperative game approach. In order to obtain a fair and efficient outcome, reference D2D pair is factored into the payoff function of each D2D pair. Sufficient conditions for the existence and uniqueness of the $\mathrm{NE}$ as well as the convergence of the proposed algorithm to the NE are provided using VI theory. Our proposed algorithm is network-assisted distributed and only requires assistance from the network in updating the reuse price and broadcasting the reference $\mathrm{D} 2 \mathrm{D}$ pair; hence it is potential for a practical design. Finally, simulation results are given to demonstrate the superiority of the proposed CLPM and FIWA.

\section{Appendix}

In Appendix, we present the proof of Theorem 7. As shown in [28], $\operatorname{VI}(\widehat{\mathscr{P}}, \mathbf{F})$ has unique solution and the mapping $\mathbf{F}$ has strong monotonicity on $\widehat{\mathscr{P}}$. Let $\mathbf{P}=\left(\mathbf{p}_{\mathbf{d}}\right)_{d=1}^{D}$ and $\mathbf{P}^{\prime}=\left(\mathbf{p}_{\mathbf{d}}^{\prime}\right)_{d=1}^{D}$; the mapping $\mathbf{F}$ is said to have strong monotonicity on $\widehat{\mathscr{P}}_{\text {if a }}$ constant $\alpha>0$ exists such that

$$
\left(\mathbf{p}_{\mathbf{d}}-\mathbf{p}_{\mathbf{d}}^{\prime}\right)^{T}\left(\mathbf{F}_{d}(\mathbf{P})-\mathbf{F}_{d}\left(\mathbf{P}^{\prime}\right)\right) \geq \alpha\left\|\mathbf{p}_{\mathbf{d}}-\mathbf{p}_{\mathbf{d}}^{\prime}\right\|^{2} .
$$

We now define

$$
\begin{aligned}
\varphi_{d}^{n} \triangleq & \sqrt{P_{d}^{n}+\sum_{j=1, j \neq d}^{D} p_{j}^{n} \bar{g}_{j, d}^{n}+\bar{\sigma}_{d}^{n}} \\
& \times \sqrt{\left(P_{d}^{n}\right)^{\prime}+\sum_{j=1, j \neq d}^{D}\left(p_{j}^{n}\right)^{\prime} \bar{g}_{j, d}^{n}+\bar{\sigma}_{d}^{n},} \\
P_{d}^{n} \triangleq & \frac{P_{d}^{n}-\left(p_{j}^{n}\right)^{\prime}}{\varphi_{d}^{n}} .
\end{aligned}
$$

From (A.1), we have the following derivation:

$$
\begin{aligned}
\left(\mathbf{p}_{\mathbf{d}}-\mathbf{p}_{\mathbf{d}}^{\prime}\right)^{T}\left(\mathbf{F}_{d}(\mathbf{P})-\mathbf{F}_{d}\left(\mathbf{P}^{\prime}\right)\right) \\
=\sum_{d=1}^{D} \sum_{n=1}^{N} \frac{p_{d}^{n}-\left(p_{j}^{n}\right)^{\prime}}{\varphi_{d}^{n}} \\
\quad \times\left(\frac{\left(p_{d}^{n}\right)-\left(p_{j}^{n}\right)^{\prime}}{\varphi_{d}^{n}}+\frac{\sum_{j \neq d} \bar{g}_{j, d}^{n}\left(p_{d}^{n}-\left(p_{j}^{n}\right)^{\prime}\right)}{\varphi_{d}^{n}}\right) \\
\geq \sum_{d=1}^{D}\left(\sum_{n=1}^{N}\left(p_{d}^{n}\right)^{2}-\sum_{j \neq d}\left|\sum_{n=1}^{N} p_{d}^{n} \frac{\bar{g}_{j, d}^{n} \varphi_{j}^{n}}{\varphi_{d}^{n}} p_{j}^{n}\right|\right)
\end{aligned}
$$

$$
\begin{aligned}
& \geq \sum_{d=1}^{D}\left(\sum_{n=1}^{N}\left(p_{d}^{n}\right)^{2}-\sum_{j \neq d}\left(\widehat{p}_{d} \max _{1 \leq n \leq N}\left(\frac{\bar{g}_{j, d}^{n} \varphi_{j}^{n}}{\varphi_{d}^{n}}\right) \widehat{p}_{j}\right)\right) \\
& \geq \sum_{d=1}^{D}\left(\widehat{p}_{d} \sum_{j=1}^{D}[\mathbf{A}]_{j, d} \widehat{p}_{j}\right)=\widehat{\mathbf{p}}^{T} \mathbf{A} \widehat{\mathbf{p}}
\end{aligned}
$$

with

$$
\begin{aligned}
\widehat{p}_{d} & =\left(\sum_{n=1}^{N}\left(P_{d}^{n}\right)^{2}\right)^{1 / 2}, \\
\widehat{\mathbf{p}} & =\left[\widehat{p}_{d}\right]_{d=1}^{D},
\end{aligned}
$$

where (A.3) follows Cauchy-Shwarz inequality. Since matrix A is PD and also a P-matrix [29], it follows from Theorem 3.3.4(b) in [34] and Lemma 2 in [29] that the constant $c(\mathbf{A}) \triangleq \min _{\|x\|_{2}=1}\left\{\max _{1 \leq d \leq D}\left\{x_{d}(\mathbf{A x})_{d}\right\}\right\}$ is positive. Also

$$
\max _{1 \leq d \leq D} x_{d}(\mathbf{A x})_{d} \geq c(\mathbf{A})\|x\|_{2}^{2}
$$

equation (A.5) holds for an arbitrary $x \in R^{D}$. At this point, joining (A.4) and (A.5) and summing over all $d$, we obtained

$$
\begin{aligned}
& \left(\mathbf{P}-\mathbf{P}^{\prime}\right)^{T}\left(\mathbf{F}(\mathbf{P})-\mathbf{F}\left(\mathbf{P}^{\prime}\right)\right) \\
& \geq \frac{\eta_{\min }(\mathbf{A})}{\max _{1 \leq d \leq \mathscr{D}} \max _{1 \leq n \leq N}\left(\varphi_{d, \max }^{n}\right)^{2}}\left\|\mathbf{P}-\mathbf{P}^{\prime}\right\|_{2}^{2},
\end{aligned}
$$

where

$$
\varphi_{d, \max }^{n}=p_{d, \max }^{n}+\left(\sum_{j=1, j \neq d}^{D} p_{d, \max }^{n} \bar{g}_{j, d}^{n}+\bar{\sigma}_{d}^{n}\right),
$$

and $n_{\min }(\mathbf{A})>0$ representing the smallest eigenvalue in $\mathbf{A}$. From the last inequality (A.6) it can be verified that strong monotonicity property of $\mathbf{F}$ exists which ends the proof.

\section{Notations}

$\mathscr{D}: \quad$ Set of D2D pairs

$\mathscr{C}$ : $\quad$ Set of cellular users

$N$ : Number of subchannels

$\mathcal{N}(c)$ : Set of subchannels assigned to a cellular user

$\tau$ : $\quad$ Iteration index

$L^{\text {max }}$ : Maximum number of iterations

$R_{d \text {,ref }}$ : Data of reference D2D pair

$r_{c}^{\mathrm{min}}$ : Rate requirement of cellular users

$u_{d}$ : Reward of selfish D2D pairs

$U_{d}$ : Payoff function of socially aware D2D pairs

$\mathscr{P}_{d}: \quad$ Strategy set of D2D pairs

$p_{d}^{n}$ : $\quad$ Transmit power of D2D pairs $d$ on subchannel $n$

$p_{c}^{n}$ : $\quad$ Transmit power of cellular users $c$ on subchannel $n$

$p_{\text {ref }}^{n}$ : Transmit power of reference D2D pair ref $_{d}^{n}$ on subchannel $n$

$p_{d, \text { max }}^{n}$ : Allowable transmit power of D2D pairs $d$ on subchannel $n$

$p_{c, \text { max }}^{n}:$ Allowable transmit power of cellular users $c$ on subchannel $n$ 
$P_{d \text {,max }}$ : Transmit power budget of D2D pairs

$P_{c, \text { max }}$ : Transmit power budget of cellular users

$\mathbf{p}_{d}$ : $\quad$ Power allocation vector for D2D pairs

$\mathbf{p}_{c}$ : $\quad$ Power allocation vector for cellular users

$\boldsymbol{\theta}$ : Cross-tier interference price

$\mathbf{t}_{d}: \quad$ Cotier interference taxation term

$J: \quad$ Jain's fairness measure

$\sigma^{2}: \quad$ Noise variance

$\bar{\sigma}_{d}^{2}$ : Normalized noise power

$\bar{g}_{j, d}^{n}$ : Normalized link gain from $\mathrm{D}_{2} \mathrm{DT}_{j}$ to $\mathrm{D}_{2} \mathrm{DR}_{d}$

$\bar{g}_{r, d}^{n}: \quad$ Normalized link gain from $\mathrm{D}_{2} \mathrm{DT}_{r}$ to $\mathrm{D}_{2} \mathrm{DR}_{d}$.

\section{Competing Interests}

The authors declare that they have no competing interests.

\section{Acknowledgments}

This work was supported by the 2016 Inje University research grant.

\section{References}

[1] D. Astely, E. Dahlman, G. Fodor, S. Parkvall, and J. Sachs, "LTE release 12 and beyond," IEEE Communications Magazine, vol. 51, no. 7, pp. 154-160, 2013.

[2] K. Doppler, M. Rinne, C. Wijting, C. B. Ribeiro, and K. Hug, "Device-to-device communication as an underlay to LTEadvanced networks," IEEE Communications Magazine, vol. 47, no. 12, pp. 42-49, 2009.

[3] A. B. MacKenzie and L. A. DaSilva, Game Theory for Wireless Engineers, vol. 1, Morgan \& Claypool Publishers, 2006.

[4] R. Cendrillon, J. Huang, M. Chiang, and M. Moonen, "Autonomous spectrum balancing for digital subscriber lines," IEEE Transactions on Signal Processing, vol. 55, no. 8, pp. 42414257, 2007.

[5] G. Scutari, D. Palomar, F. Facchinei, and J.-S. Pang, "Convex optimization, game theory, and variational inequality theory," IEEE Signal Processing Magazine, vol. 27, no. 3, pp. 35-49, 2010.

[6] R. Yin, G. Yu, H. Zhang, Z. Zhang, and G. Y. Li, "Pricing-based interference coordination for $\mathrm{d} 2 \mathrm{~d}$ communications in cellular networks," IEEE Transactions on Wireless Communications, vol. 14, no. 3, pp. 1519-1532, 2015.

[7] G. Yu, L. Xu, D. Feng, R. Yin, G. Y. Li, and Y. Jiang, "Joint mode selection and resource allocation for device-to-device communications," IEEE Transactions on Communications, vol. 62, no. 11, pp. 3814-3824, 2014.

[8] C.-H. Yu, K. Doppler, C. B. Ribeiro, and O. Tirkkonen, "Resource sharing optimization for device-to-device communication underlaying cellular networks," IEEE Transactions on Wireless Communications, vol. 10, no. 8, pp. 2752-2763, 2011.

[9] H. Min, J. Lee, S. Park, and D. Hong, "Capacity enhancement using an interference limited area for device-to-device uplink underlaying cellular networks," IEEE Transactions on Wireless Communications, vol. 10, no. 12, pp. 3995-4000, 2011.

[10] M. Zulhasnine, C. Huang, and A. Srinivasan, "Efficient resource allocation for device-to-device communication underlaying
LTE network," in Proceedings of the 6th Annual IEEE International Conference on Wireless and Mobile Computing, Networking and Communications (WiMob '10), pp. 368-375, Niagara Falls, Canada, October 2010.

[11] J. Zhao, K. K. Chai, Y. Chen, J. Schormans, and J. Alonso-Zarate, "Joint mode selection and resource allocation for machinetype D2D links," Transactions on Emerging Telecommunications Technologies, 2015.

[12] D. Feng, L. Lu, Y. Yuan-Wu, G. Y. Li, G. Feng, and S. Li, "Device-to-device communications underlaying cellular networks," IEEE Transactions on Communications, vol. 61, no. 8, pp. 3541-3551, 2013.

[13] Y. Li, D. Jin, J. Yuan, and Z. Han, “Coalitional games for resource allocation in the device-to-device uplink underlaying cellular networks," IEEE Transactions on Wireless Communications, vol. 13, no. 7, pp. 3965-3977, 2014.

[14] Y. Zhang, C.-Y. Wang, and H.-Y. Wei, "Incentive compatible mode selection and spectrum partitioning in overlay D2Denabled network," in Proceedings of the IEEE Globecom Workshops (GC Wkshps '15), pp. 1-6, IEEE, San Diego, Calif, USA, December 2015.

[15] Y. Liu and S. Feng, "Interference pricing for device-to-device communications," in Proceedings of the 1st IEEE International Conference on Communications (ICC '14), Sydney, Australia, June 2014.

[16] C. Sun, M. Peng, Y. Sun, Y. Li, and J. Jiang, "Distributed power control for device-to-device network using stackelberg game," in Proceedings of the IEEE Wireless Communications and Networking Conference (WCNC '14), pp. 1344-1349, IEEE, Istanbul, Turkey, April 2014.

[17] C. Xu, L. Song, Z. Han et al., "Efficiency resource allocation for device-to-device underlay communication systems: a reverse iterative combinatorial auction based approach," IEEE Journal on Selected Areas in Communications, vol. 31, no. 9, pp. 348-358, 2013.

[18] C. Xia, S. Xu, and K. S. Kwak, "Overlapping coalition formation games-based resource allocation for device-to-device communication in LTE-A network," Transactions on Emerging Telecommunications Technologies, vol. 26, no. 12, pp. 1295-1305, 2015.

[19] F. Hajiaghajani, R. Davoudi, and M. Rasti, "A joint channel and power allocation scheme for Device-to-Device communications underlaying uplink cellular networks," in Proceedings of the IEEE Conference on Computer Communications Workshops (INFOCOM WKSHPS '16), pp. 768-773, IEEE, San Francisco, Calif, USA, April 2016.

[20] Y. Liu, R. Wang, and Z. Han, "Interference-constrained pricing for D2D networks," IEEE Transactions on Wireless Communications, vol. PP, no. 99, 2016.

[21] Y. Kai and H. Zhu, "Resource allocation for multiple-pair D2D communications in cellular networks," in Proceedings of the IEEE International Conference on Communications (ICC '15), pp. 2955-2960, London, UK, June 2015.

[22] F. Wang, L. Song, Z. Han, Q. Zhao, and X. Wang, "Joint scheduling and resource allocation for device-to-device underlay communication," in Proceedings of the IEEE Wireless Communications and Networking Conference (WCNC '13), pp. 134139, IEEE, Shanghai, China, April 2013.

[23] R. Yin, C. Zhong, G. Yu, Z. Zhang, K. K. Wong, and X. Chen, "Joint spectrum and power allocation for $\mathrm{d} 2 \mathrm{~d}$ communications underlaying cellular networks," IEEE Transactions on Vehicular Technology, vol. 65, no. 4, pp. 2182-2195, 2016. 
[24] H.-H. Nguyen, M. Hasegawa, and W.-J. Hwang, "Distributed resource allocation for D2D communications underlay cellular networks," IEEE Communications Letters, vol. 20, no. 5, pp. 942945, 2016.

[25] J. Winters, "Smart antenna techniques and their application to wireless ad hoc networks," IEEE Wireless Communications, vol. 13, no. 4, pp. 77-83, 2006.

[26] H. Hindi, "A tutorial on convex optimization," in Proceedings of the American Control Conference (AAC '04), vol. 4, pp. 32523265, July 2004.

[27] G. Scutari, D. P. Palomar, and S. Barbarossa, "Asynchronous iterative water-filling for Gaussian frequency-selective interference channels," IEEE Transactions on Information Theory, vol. 54, no. 7, pp. 2868-2878, 2008.

[28] J.-S. Pang, G. Scutari, D. P. Palomar, and F. Facchinei, “Design of cognitive radio systems under temperature-interference constraints: a variational inequality approach," IEEE Transactions on Signal Processing, vol. 58, no. 6, pp. 3251-3271, 2010.

[29] G. Scutari, D. P. Palomar, and S. Barbarossa, "Optimal linear precoding strategies for wideband noncooperative systems based on game theory-part I: nash equilibria," IEEE Transactions on Signal Processing, vol. 56, no. 3, pp. 1230-1249, 2008.

[30] R. A. Horn and C. R. Johnson, Matrix Analysis, Cambridge University Press, Cambridge, UK, 1985.

[31] 3GPP, "Simulation assumptions and parameters for FDD HeNB RF requirements," 3GPP R4-092042, 2010.

[32] J. Papandriopoulos and J. S. Evans, "Low-complexity distributed algorithms for spectrum balancing in multi-user DSL networks," in Proceedings of the IEEE International Conference on Communications (ICC '06), vol. 7, pp. 3270-3275, Istanbul, Turkey, July 2006.

[33] R. K. Jain, D. W. Chiu, and W. R. Hawe, A Quanlitative Measure of Fairness and Discrimination for Resource Allocation in a Shared Computer Systems, Digital Equipment Corp, Hudson, Mass, USA, 1984, http://www.cs.wustl.edu/ jain/papers/ftp/ fairness.pdf.

[34] R. W. Cottle, J.-S. Pang, and R. E. Stone, The linear complementarity problem, vol. 60 of Classics in Applied Mathematics, 2009. 

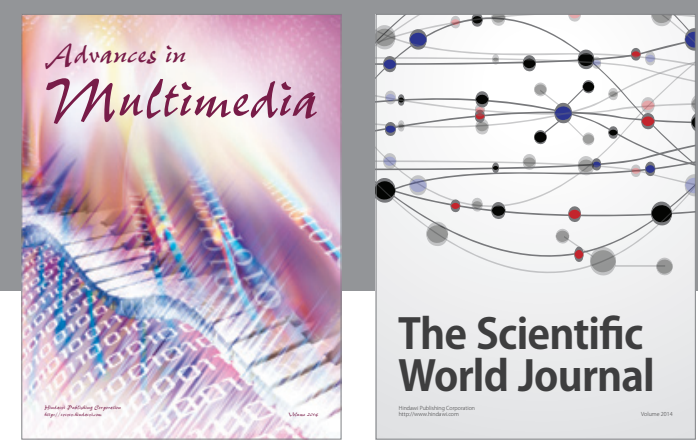

The Scientific World Journal
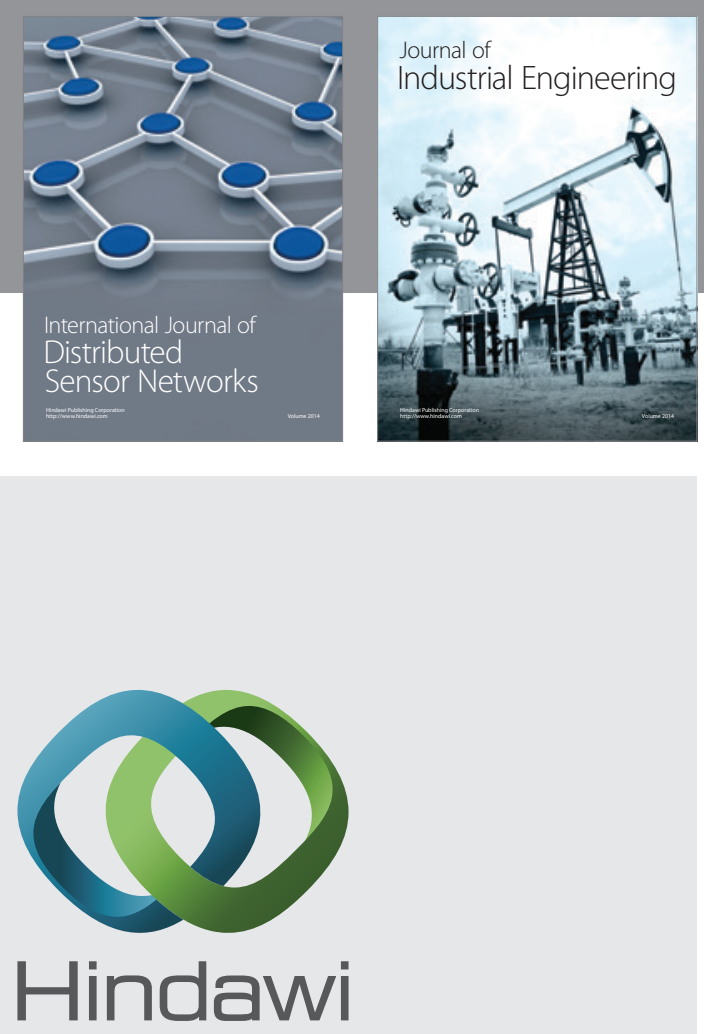

Submit your manuscripts at

https://www.hindawi.com

\section{Computer Networks} and Communications
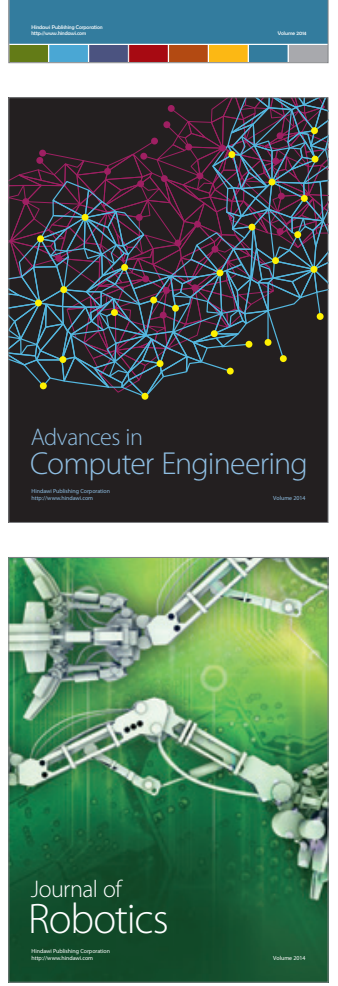
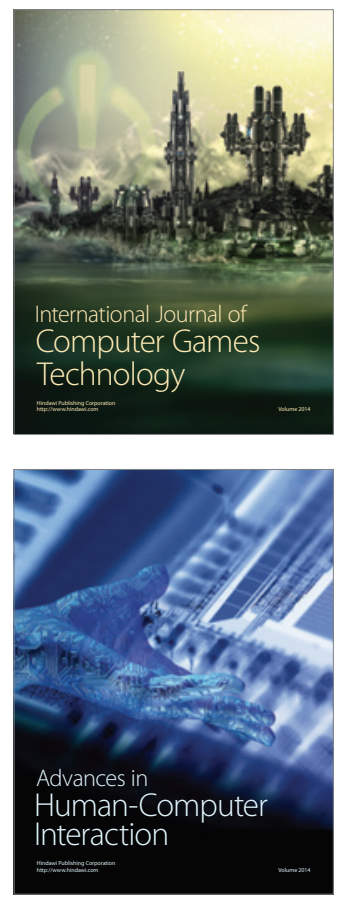
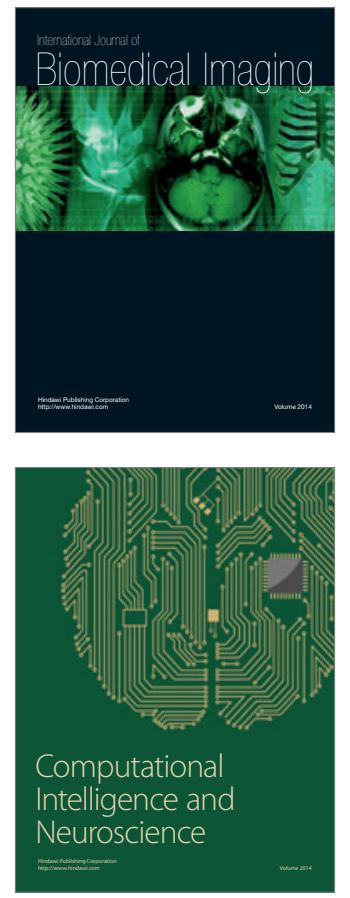
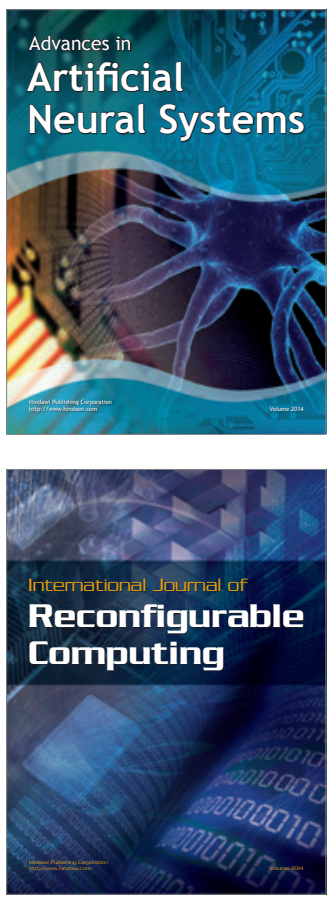
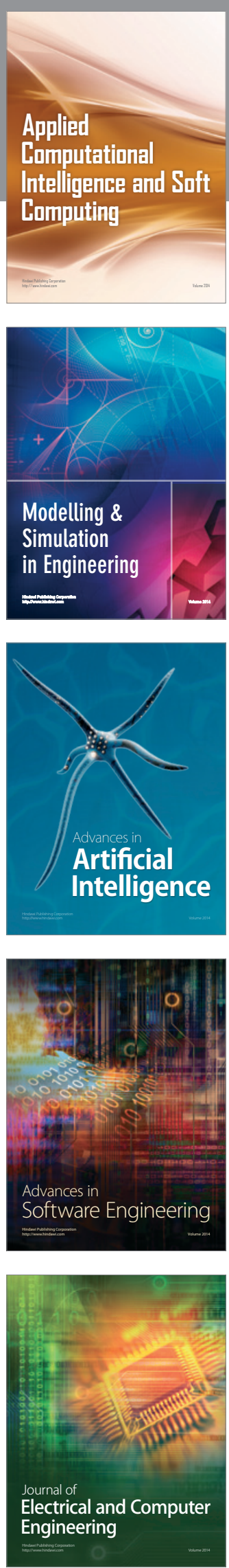